\title{
Human Body Shadowing Effect on Dynamic UWB On-Body Radio Channels
}

\author{
Timo Kumpuniemi, Student Member, IEEE, Matti Hämäläinen, Senior Member, IEEE, \\ Kamya Yekeh Yazdandoost, Senior Member, IEEE, and Jari Iinatti, Senior Member, IEEE
}

\begin{abstract}
This letter reports the shadowing effects of a human body on dynamic ultra-wideband on-body radio links. A vector network analyzer is used to sweep the 3-5 GHz frequency band in an anechoic chamber. The sweep time is set fast enough to fulfil the channel coherence time requirements. This enables the data analysis to be performed in time domain by examining the corresponding channel impulse responses. Five on-body locations are examined: right wrist (RW), left wrist with an antenna on the palm (LWA) or back side (LWB) of the hand, left arm (AL) and left ankle (LA). Two antenna types are applied: dipole and double loop. The path loss (PL) lies between 35.9-85.8 dB depending on the case. The double loop has, on the average, higher PL than the dipole. The LWA-RW and RW-LA links have the lowest and highest PLs, respectively. The amplitudes of the first arriving paths follow the inverse Gaussian distribution. The level crossing rate (LCR) and the average fade duration (AFD) show weaker performance for the double loop antennas compared to the dipoles. In general, the LWA-LA is the poorest link with respect to the LCR and the AFD.
\end{abstract}

Index Terms-Ultra-wideband (UWB), on-body, dynamic channel, human body, shadowing.

\section{INTRODUCTION}

$\mathrm{T}$ HE decreasing sizes of electronic devices integrated with wireless technology, in addition to increases in the efficiency of computational processing and energy consumption, have enabled their usage in new environments such as the close vicinity of the human body when carried in pockets or attached otherwise on the body as sensors. Populations are ageing in many countries due to increasing life expectancies [1]. At the same time, chronic diseases are increasing [2]. These megatrends together with the worldwide interest boost in a person's own wellbeing have all rapidly speeded up the activity in the field of wireless body area networks (WBANs). The sensors in WBANs can be attached on the body surface (on-body), close to the body (off-body) or inside it (in-body) [3]. In medicine, WBANs raise the quality of life of the patients as their health can be, e.g., monitored remotely with various sensors while living in their homes

Manuscript received March 1, 2016; resubmitted October 21, 2016; accepted January 7, 2017. The work was funded in part by the Finnish Funding Agency for Innovation (Tekes) by the project Wireless Body Area Network for Health and Medical Care (WiBAN-HAM).

The authors are with the Centre for Wireless Communications, P.O. Box 4500, FI-90014 University of Oulu, Finland. (E-mails: \{timo.kumpuniemi matti.hamalainen|jari.iinatti\}@oulu.fi, yazdandoost@ieee.org). instead of locating them in medical institutions. The workload of the medical staff is eased up increasing the cost-efficiency of the medical care. In sports and wellbeing, the individuals can utilize WBANs in, e.g., following the effectiveness of their training or their daily physical activity or the quality of sleep. The communication distances between the sensors and nodes collecting their data are typically short in WBANs. Thus the ultra-wideband (UWB) technology is very suitable for them offering also a low transmission power with a low interference and a power consumption and a high positioning accuracy [4]. In 2012, the IEEE published the standard IEEE802.15.6 [5] for WBANs where UWB is defined as one physical layer technique to be used at the frequency ranges of 3.2448-4.7424 GHz and 6.2400-10.2336 GHz. For this reason, knowledge about the behavior of WBAN UWB radio channels is needed.

References for both off-body and on-body UWB channel modeling exist. In [6], static off-body measurements are reported. In [7], dynamic off-body measurements are carried out with a vector network analyzer (VNA) in an $800 \mathrm{MHz}$ bandwidth. Static off- and on-body UWB channels are reported widely in [8]. Pseudo-dynamic UWB on-body channel modeling is examined in [9] and dynamic UWB onbody measurements in the time domain in [10]. In [11], dynamic UWB on-body channels are measured by frequency sweeps with a VNA although the scope is not on the channels.

The human body shadowing in on-body channels is noted to be considerable both with narrowband signals in $[12,13]$ and with an UWB signal in [9]. However, to the best of our knowledge, no one has reported the human body shadowing effects on dynamic on-body UWB channels with VNA measurements by examining the channel impulse responses (CIRs). With the CIR approach the energy contained in each CIR tap can be solved and be used, e.g., in a RAKE receiver design. A VNA offers, e.g., a high dynamic range of the CIRs for the data analysis. A disadvantage is that both antennas must be attached to the VNA which reduces the mobility. Also the sweeping nature of the VNA sets a limit to the maximum velocities possible to reliably observe in the channel.

This work applies VNA frequency domain measurements in the $3-5 \mathrm{GHz}$ frequency band joined with the data analysis with the CIRs. The anechoic chamber environment excludes the reflected paths originating beyond the human body. The measurements are repeated with two planar prototype antenna types, dipole and double loop. 


\section{Measurement SetuP}

A $183 \mathrm{~cm}$ tall with $95 \mathrm{~kg}$ of weight male test subject at his late twenties was wearing a normal T-shirt and jeans. Shoes and all metal containing items were removed. The $245 \mathrm{~cm}$ by $365 \mathrm{~cm}$ large anechoic chamber was built of movable absorber blocks and it was situated inside an electromagnetic compatibility room with a floor area of $60 \mathrm{~m}^{2}$.

A four port VNA (Rohde \& Schwarz ZVA8) was located outside the chamber. The VNA was set to sweep the $3-5 \mathrm{GHz}$ frequency band with 201 points with a sweep time of 28.944 ms. During one measurement 500 sweeps were recorded. All sixteen scattering parameter were measured. The VNA transmit power was set to $+10 \mathrm{dBm}$ and the VNA intermediate frequency filter bandwidth to $1 \mathrm{MHz}$.

Four antennas at a time were connected to the VNA by using eight meter long armored measurement cables. The dimensions, performances and radiation patterns of the planar prototype antenna types (dipole and double loop) are described in details in [14-16].

\section{MEASURED SCENARIOS}

Five antenna locations were selected on the body as shown in Fig. 1a. They are the right wrist (RW) with the antenna attached on the palm side of the wrist, the left wrist with the antenna at the palm side (LWA), the left wrist with the antenna at the side of the back of the hand (LWB), the left arm (AL) and the left ankle (LA). At the AL and LA the antenna is attached at the lateral side of the arm or ankle. The antennas were separated from the body with a $20 \mathrm{~mm}$ thick piece of ROHACELL $31 \mathrm{HF}$ with electrical properties corresponding to air. The $20 \mathrm{~mm}$ distance is found to be a balanced compromise for simultaneously providing good antenna match and acceptable channel path gains [14,15]. The links LWARW, LWA-LA, RW-LA and LWB-AL were measured twice in both forward and reverse direction for both antenna types. As a result, 2000 sweeps are recorded for each link.

During a measurement the test subject was emulating a walk of an elderly person by walking in place at a pace of approx. 0.7 steps per second for 54 seconds. The maximum trajectory length of an antenna site during one step is approx. $25 \mathrm{~cm}$ corresponding to a velocity of $0.71 / \mathrm{s} \cdot 0.25 \mathrm{~m}=0.175$ $\mathrm{m} / \mathrm{s}$. The relative theoretical maximum velocity between an antenna pair is $0.35 \mathrm{~m} / \mathrm{s}$. The maximum Doppler frequency can be solved as [17]

$$
f_{d}=v / \lambda
$$

where $v$ is the relative velocity and $\lambda$ is the signal wavelength. At $5 \mathrm{GHz}, f_{d}$ is thus $5.8 \mathrm{~Hz}(3.5 \mathrm{~Hz}$ at $3 \mathrm{GHz})$. Several equations are proposed in [17] to evaluate the resulting coherence time $T_{0}$ of a fading channel. The most stringent demand is set by

$$
T_{0}=9 /\left(16 \pi f_{d}\right)
$$

It results $T_{0}=30.8 \mathrm{~ms}$ at $5 \mathrm{GHz}(51.1 \mathrm{~ms}$ at $3 \mathrm{GHz})$ exceeding

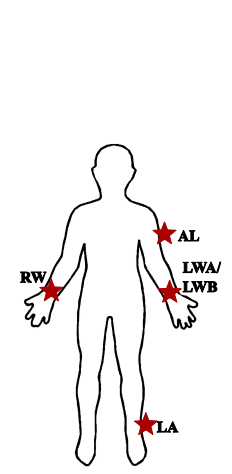

a)
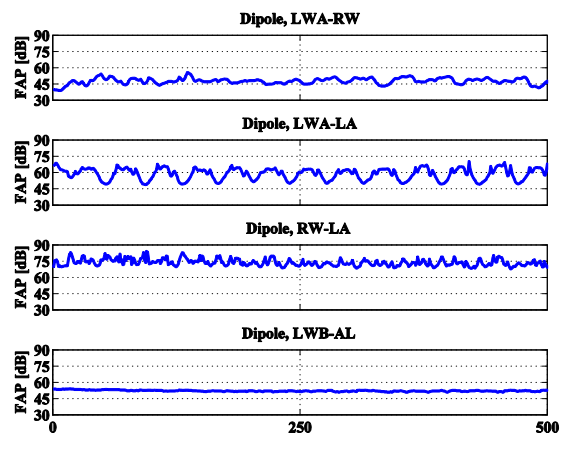

b)

Fig. 1. a) The selected antenna locations b) The PLs of the FAPs of the links in the dipole antenna case.

the used sweep time of $28.944 \mathrm{~ms}$. Thus the demand for a channel to be constant during one sweep is fulfilled.

\section{RESUlts}

\section{A. Data Processing}

The inverse fast Fourier transform algorithm is applied to the measured complex frequency domain data to extract the corresponding complex valued CIRs. No windowing is used. The absolute values of the CIRs are then used for the analysis.

\section{B. Path Loss}

The path loss (PL) of the first arriving paths (FAPs) as a function of first 500 sweeps, i.e., time for each link is shown in the case of the dipole antenna in Fig. 1b. A FAP is solved by finding the first value where the slope of the CIR changes from positive to negative. The value must be above the threshold set to be $6 \mathrm{~dB}$ higher than the mean of the 30 first linear valued CIR samples. It can be directly noted that the characteristics of the links vary greatly both in the level of the PL and its variation. The LWB-AL link has visually observed the lowest loss and variation rate whereas the RW-LA link has the highest loss and variation rate.

The average PL, $\mu_{\mathrm{PL}}$, is calculated from the FAPs of the linear valued CIRs. The maximum value of the PL, $P L_{\mathrm{V}}$, and the minimum value, $P L_{\wedge}$, are solved as well. The standard deviation of the PL, $\sigma_{\mathrm{PL}}$, is extracted from the decibel valued FAPs. The results are presented for each link. This is repeated for both antenna types. The results are collected in Table I. The PLs vary between $35.9-84.2 \mathrm{~dB}$ and $37.4-85.8 \mathrm{~dB}$ with the dipole and the double loop, respectively. LWA-RW is the best link having the lowest $\mu_{\mathrm{PL}}$. The signal propagates in LWA-RW mostly in air whereas in the other links the creeping wave propagation is more or less present explaining the result. The RW-LA link has the highest $\mu_{\mathrm{PL}}$. The LWA-LA has the largest $\sigma_{\mathrm{PL}}$ among the separate links. The most stabile link with the lowest $\sigma_{\mathrm{PL}}$ is the LWB-AL since the relative positions of the antennas do not vary much. The double loop antenna produces higher $\sigma_{\mathrm{PL}}$ values in all links.

The statistical amplitude distributions are found out by fitting the linear valued FAPs to 17 continuous distributions: beta, Birnbaum-Saunders, exponential, extreme value, gamma, 
generalized extreme value, generalized Pareto, inverse Gaussian, logistic, log-logistic, lognormal, Nakagami, normal, Rayleigh, Rician, t location-scale and Weibull distributions. Unlike many articles in the field, the selection was deliberately decided to be wide and not limited into a few based on, e.g., easily assumed physical explanations of the scenario. The distributions are ranked according to the second order Akaike information criterion [18], recommended in $[18,19]$. The best fit in all cases is obtained by the inverse Gaussian distribution with the probability density function of [20]

$$
f(x \mid \alpha, \beta)=\sqrt{\frac{\beta}{2 \pi x^{3}}} \exp \left\{-\frac{\beta}{2 \alpha^{2} x}(x-\alpha)^{2}\right\}, x>0,
$$

where $\alpha>0$ and $\beta>0$ are the mean and shape parameters. The distribution parameters are collected in Table II.

To verify the distribution model, probability density functions (PDFs) for the dipole case are shown in Fig. 2. The curves based on empirical (emp.) data and modeled (mod.) data follow each other closely. In LWA-LA two maximums exist in the empirical data indicating that for a higher accuracy a mixed distribution model should be used with the cost of increased complexity.

\section{Level Crossing Rate and Average Fade Duration}

The level crossing rate (LCR) describes the number of positive-going crossings of a signal in unit time and the average fade duration (AFD) the average time between the negative and positive zero-crossings [21]. They give valuable information of the characteristics of a fading channel for system design purposes. The measured FAPs of all links are normalized by their root-mean-square values in order to extract the LCR and AFD.

Fig. 3 presents the LCR in hertz for both antennas. They are calculated by dividing the number of positive-going threshold level crossings by the total time of the measurements, i.e. four times $54 \mathrm{~s}$ resulting $216 \mathrm{~s}$. The $\mathrm{x}$-axis describes the normalized FAP level denoted by $\rho=R / r_{\text {rms }}$, where $R$ is the threshold level and $r_{\text {rms }}$ is the root-mean-square value of the FAP sample train. The RW-LA has the highest LCR correlating with the visual observation of Fig. 1b. The LWA-LA curve has the widest variation of $\rho$ expressing the largest variation around the rms value. The LWB-AL has a very narrow LCR curve reflecting the higher stability of the channel as seen in Fig. 1b and Table I. The LCR curves extend to smaller values of $\rho$ for the double loop case compared to the dipole indicating the different characteristics, e.g., in the radiation patterns of the antennas. The LCRs are low in frequency as the slow walking pace of the test subject. However, the walking is a cyclic movement thus enabling the scaling of the LCR and AFD results for faster walking paces.

Fig. 4 presents the AFD for both antennas. The LWA-LA has the highest AFD at $\rho[\mathrm{dB}]<0 \mathrm{~dB}$. Due to the stable nature of the LWB-AL, it has a rapidly growing AFD curve saturating at lower $\rho[\mathrm{dB}]$ value than the other curves. The
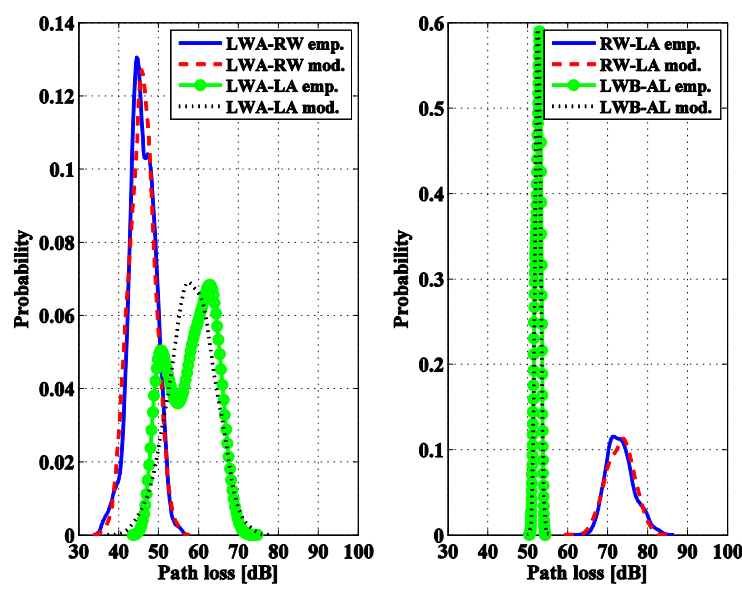

Fig. 2. The empirical and modeled PDFs in the dipole antenna case.

TABLE I



\begin{tabular}{lcccccccc}
\hline \hline & \multicolumn{4}{c}{ Dipole } & \multicolumn{5}{c}{ Double Loop } \\
\hline \multirow{2}{*}{ Link } & $\begin{array}{c}\mu_{\mathrm{PL}} \\
{[\mathrm{dB}]}\end{array}$ & $\begin{array}{c}\sigma_{\mathrm{PL}} \\
{[\mathrm{dB}]}\end{array}$ & $\begin{array}{c}P L_{\vee} \\
{[\mathrm{dB}]}\end{array}$ & $\begin{array}{c}P L_{\wedge} \\
{[\mathrm{dB}]}\end{array}$ & $\begin{array}{c}\mu_{\mathrm{PL}} \\
{[\mathrm{dB}]}\end{array}$ & $\begin{array}{c}\sigma_{\mathrm{PL}} \\
{[\mathrm{dB}]}\end{array}$ & $\begin{array}{c}P L_{\vee} \\
{[\mathrm{dB}]}\end{array}$ & $P L_{\wedge}$ \\
& {$[\mathrm{dB}]$} \\
\hline LWA-RW & 45.3 & 3.1 & 55.4 & 35.9 & 49.0 & 4.9 & 69.2 & 37.4 \\
LWA-LA & 56.4 & 5.6 & 70.1 & 48.1 & 57.2 & 9.6 & 85.5 & 49.9 \\
RW-LA & 72.5 & 3.4 & 84.2 & 63.9 & 69.6 & 5.3 & 85.8 & 60.2 \\
LWB-AL & 52.5 & 0.7 & 53.9 & 50.8 & 56.3 & 1.3 & 60.9 & 51.9 \\
\hline \hline
\end{tabular}

TABLE II

PARAMETERS FOR STATISTICAL AMPLITUDE DisTRIBUTIONS

\begin{tabular}{lcccc}
\hline \hline & \multicolumn{2}{c}{ Dipole } & \multicolumn{2}{c}{ Double Loop } \\
\hline Link & $\alpha$ & $\beta$ & $\alpha$ & $\beta$ \\
\hline LWA-RW & $54.21 \cdot 10^{-4}$ & $386.02 \cdot 10^{-4}$ & $35.36 \cdot 10^{-4}$ & $93.40 \cdot 10^{-4}$ \\
LWA-LA & $15.10 \cdot 10^{-4}$ & $30.42 \cdot 10^{-4}$ & $13.74 \cdot 10^{-4}$ & $6.65 \cdot 10^{-4}$ \\
RW-LA & $2.36 \cdot 10^{-4}$ & $14.34 \cdot 10^{-4}$ & $3.29 \cdot 10^{-4}$ & $7.42 \cdot 10^{-4}$ \\
LWB-AL & $23.64 \cdot 10^{-4}$ & $4155.97 \cdot 10^{-4}$ & $15.39 \cdot 10^{-4}$ & $697.70 \cdot 10^{-4}$ \\
\hline \hline
\end{tabular}
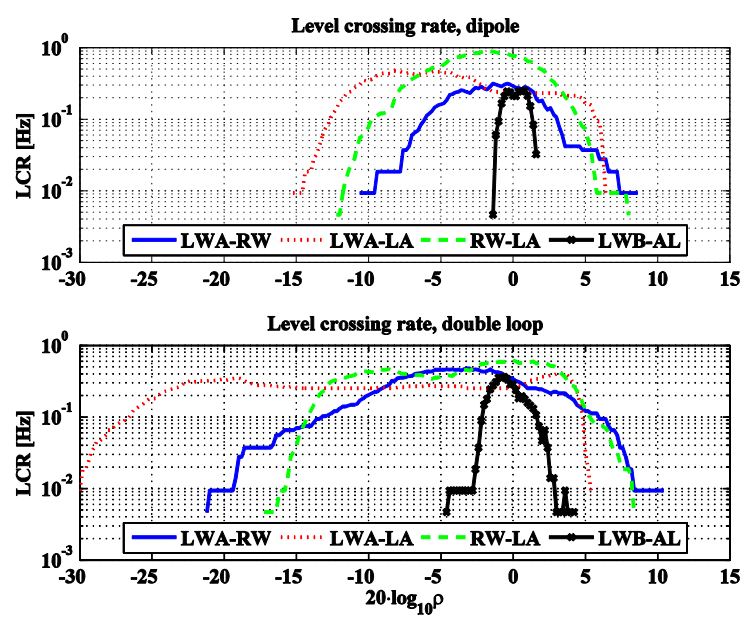

Fig. 3. The level crossing rates of the links.

saturation value corresponds to the total measurement time.

The double loop antenna has non-zero AFD values extending to lower values of $\rho$ compared to the dipole antenna. The fading problem is thus in this sense more severe when 



Fig. 4. The average fade durations of the links.

using the double loops compared to the dipole antenna case.

The main explanation on the differences between the antennas in their performances lies in their radiation patterns [16]. The double loop has clearer nulls in its pattern. As a result, in dynamic conditions the average radiation patterns cause weaker performance on the examined parameters. On the contrary to our result, in [22] magnetic antennas, e.g. loop antennas, are preferred at the proximity of a human body. However, [22] assumes electrically small antennas. In the UWB case especially at the upper parts of the bandwidth the antenna can no longer be considered electrically small.

\section{CONCLUSION}

This letter considers human body shadowing effects in dynamic on-body UWB radio channels. The results are based on VNA measurements by sweeping a frequency band of 3-5 GHz. The work is done in an anechoic chamber in order to limit the examination to the body effects only. Two planar prototype antenna types are used: dipole and double loop. The data analysis is performed by using CIRs. The PLs vary between 35.9-85.8 $\mathrm{dB}$ depending on the link and antenna. The double loop has, on the average, a higher PL compared to the dipole. The lowest average PL has the LWA-RW whereas the RW-LA has the largest value. The highest and lowest standard deviations of the PL are noted with the LWA-LA and LWB$\mathrm{AL}$, respectively. The amplitudes of the received signals follow the inverse Gaussian distribution unlike usually seen in the references. With respect to the LCR, the RW-LA has the maximum value for both antennas but the LWA-LA has nonzero LCR values in the largest range of thresholds. The LWALA has the highest AFD below the $0 \mathrm{~dB}$ threshold, and the LWB-AL above it. The double loop has non-zero LCR and AFD values in a larger range of $\rho$ compared to the dipole. Thus the fading problem is not as difficult with the dipoles.

\section{REFERENCES}

[1] R. Lee, "The demographic transition: three centuries of fundamental change," Journal of Economic Perspectives, AEA, vol. 17, Fall 2003
[2] D. Yach, C. Hawkes, C. L. Gould, and K. J. Hofman, "The global burden of chronic diseases, overcoming impediments to prevention and control," The Journal of the American Medical Association, JAMA, vol. 291, pp. 2616-2622, June 2004.

[3] P. S. Hall, and Y. Hao, Antennas and Propagation for Body-Centric Wireless Communications, 2nd ed. Norwood, MA, Artech House, 2012, pp. 1-16.

[4] I. Oppermann, M. Hämäläinen, and J. Iinatti (eds.), UWB Theory and Applications. West Sussex, England, John Wiley \& Sons, 2004, pp. 1-8.

[5] IEEE standard for local and metropolitan area networks-Part 15.6: wireless body area networks, IEEE Standard 802.15.6-2012, 2012.

[6] R.-G. Garcia-Serna, C. Garcia-Pardo, and J. Molina-Garcia-Pardo, "Effect of the receiver attachment position on ultrawideband off-body channels," IEEE Antennas Wireless Propag. Lett., vol. 14, pp. 11011104, May 2015.

[7] A. J. Ali, W. G. Scanlon, and S. L. Cotton, "Pedestrian effects in indoor UWB off-body communication channels," in Proc. 2010 Loughborough Antennas \& Propag. Conf., 2010, pp. 57-60.

[8] R. Di Bari, Q. H. Abbasi, A. Alomainy, and Y. Hao, "An advanced UWB channel model for body-centric wireless networks," Progress In Electromagnetics Research, vol. 136, pp. 79-99, Jan. 2013.

[9] T. Kumpuniemi, M. Hämäläinen, T. Tuovinen, K. Yekeh Yazdandoost, and J. Iinatti, "Radio channel modelling for pseudo-dynamic WBAN onbody UWB links," in Proc. 8th Int. Symp. Medical Inform. and Commun. Technol. (ISMICT), 2014, pp. 1-5.

[10] A. Maskooki, C. B. Soh, E. Gunawan, and K. S. Low, "Ultra-wideband real-time dynamic channel characterization and system-level modeling for radio links in body area networks," IEEE Trans. Microw. Theory Tech., vol. 61, no. 8, pp. 2995-3004, Aug. 2013.

[11] M. Qaraqe, Q. H. Abbasi, A. Alomainy; and E. Serpedin, "Experimental evaluation of MIMO capacity for ultrawideband body-centric wireless propagation channels," IEEE Antennas Wireless Propag. Lett., vol. 13, pp. 495-498, March 2015 .

[12] R. Rosini, and R. D'Errico, "Comparing on body dynamic channels for two antenna designs," in Proc. 2012 Loughborough Antennas \& Propag. Conf., 2012, pp. 1-4.

[13] S. Cotton, "A statistical model for shadowed body-centric communications channels: theory and validation," IEEE Trans. Antennas Propag., vol. 62, no. 3, pp. 1416-1424, March 2014.

[14] T. Tuovinen, T. Kumpuniemi, K. Yekeh Yazdandoost, M. Hämäläinen, and J. Iinatti,. "Effect of the antenna-human body distance on the antenna matching in UWB WBAN applications," in Proc. 7th Int. Symp. on Medical Inform. and Commun. Technol. (ISMICT), 2013, pp. 193197.

[15] T. Tuovinen., T. Kumpuniemi, M. Hämäläinen., K. Yekeh Yazdandoost, and J. Iinatti, "Effect of the antenna-body distance on the on-ext and onon channel link path gain in UWB WBAN applications," in Proc. 35th Annu. Int. Conf. IEEE Eng. in Medicine and Biology Soc. (EMBC), 2013, pp. 1242-1245.

[16] T. Kumpuniemi, M. Hämäläinen, K. Yekeh Yazdandoost, and J. Iinatti, "Measurements for body-to-body UWB WBAN radio channels," in Proc. 9th Eur. Conf. on Antennas and Propag. (EUCAP), 2015, pp. 1-5.

[17] B. Sklar, Digital Communications, Fundamentals and Application, 2nd ed. Upper Saddle River, NJ, USA, Prentice Hall, 2001, pp. 944-1011.

[18] K. P. Burnham, and D. R. Anderson, Model Selection and Multimodel Inference, A Practical Information-Theoretic Approach, 2nd ed. New York, USA, Springer-Verlag, 2002, pp. 60-72.

[19] A. F. Molisch, K. Balakrishnan, D. Cassioli, C.-C. Chong, S. Emami, A. Fort, J. Karedal, J. Kunisch, H. Schantz, U. Schuster, and K. Siwiak, "IEEE 802.15.4a channel model-final report," IEEE 802.15.4a channel modeling subgroup, 2004, pp. 1-40.

[20] M. Rausand, and A. Høyland, System Reliability Theory, Models, Statistical Methods, and Applications. Hoboken, USA, John Wiley \& Sons, 2004, pp. 41-54.

[21] S. R. Saunders, and A. Aragón-Zavala, Antennas and Propagation for Wireless Communication Systems, 2nd ed. Chichester, West Sussex, England, John Wiley \& Sons, 2007, pp. 209-240.

[22] K. Fujimoto, and J. K. James, Mobile Antenna Systems Handbook, 2nd ed. Norwood, MA, Artech House, 2001, pp. 293-361. 